\title{
Apply a Screensaver Template for Windows 98
}

\section{Zuhor H. Hasan}

College of Administration and Economics

University of Mosul

\author{
Ahmed S. Nori \\ ahmed.s.nori \\ @uomosul.edu.iq
}

\author{
Asmaa Y. Hamo \\ asmahammo \\ @uomosul.edu.iq
}

College of Computer Science and Mathematics University of Mosul, Iraq

Received on: 16/02/2002

\section{Accepted on: 12/10/2002}

\section{ABSTRACT}

This paper involves designing graphics model for displaying and working under Windows98 operating system called Screen Saver, which is considered as one of the most significant desktop settings. Various and multiple algorithms were used and implemented for designing Screen Saver, with the capabilities of (storing its settings within the Windows98 Registry and preventing using the computer from an unauthorized user).

To perform this work, Visual C++ v6.0 and Windows98 API functions were considered as programming tools. Software was tested successfully under Windows 98 operating system.

Keywords: Screen Saver, Registry, API.

$$
\begin{aligned}
& \text { تطبيق قالب حافظة شاشة لنظام التشغيل ويندوز98 }
\end{aligned}
$$

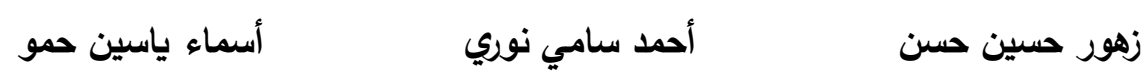

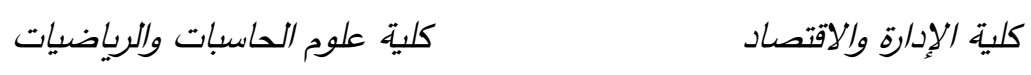

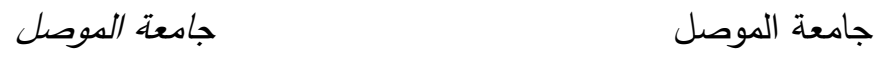

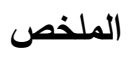


كما يستخدم القالب في حماية الحاسبة من الأشخاص غير المخولين وذلك عن طريق

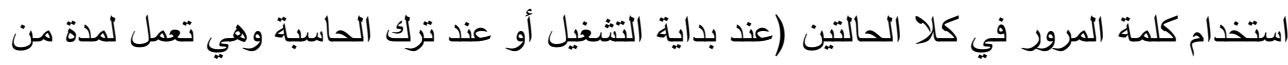
الزمن) دون استجابة من المستخدم أي (الضغط على لوحة المفاتيح أو جهاز الفأرة). استخدمت لغة Visual C++ V6.0 في البرمجة مـع الاعتماد على أسلوب واجهة البرمجة التطبيقية API وتعمل بنجاح في بيئة نظام التشغيل ويندوز 98.

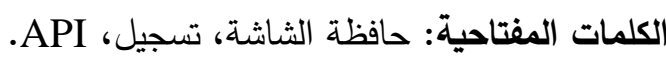

المقدمة

يرتبط نظـام التشـيل ويندوز 98 مـع المستخدم بواجهة رسومية تدعى واجهـة المستخدم الرسومية Graphic User Interface GUI باستخدام أسلوب سطح الدكتب إذ يتفاعل المستخدم مع النظام من خلال كائنات ورموز متنوعة [5].

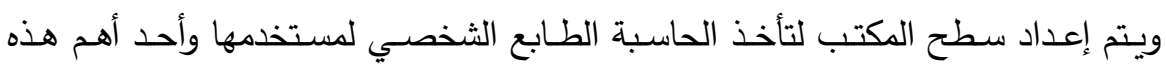
الإعدادات هو حافظة الثاشة ـ ومبدأ عمل حافظة الثاشة هو انه خلال فترة زمنية سبق أن حددها

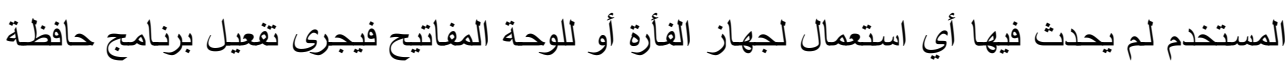

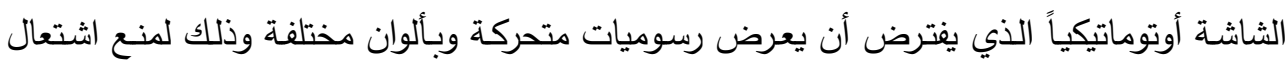

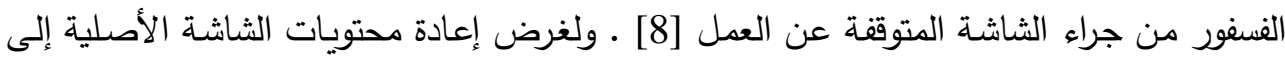
وضعها يمكن الضغط على أي مفتاح في لوحة المفاتيح أو تحريك جهاز الفأرة.

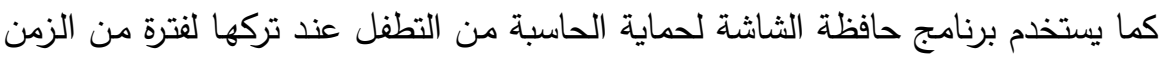
وذلك بخزن مسبق لكلمة المرور Password . بعد تشغيل برنامج حافظة الثاشة فان أي استخدام للمفاتيح أو تحريك لجهاز الفأرة لن يعيد محتويـات الثاشـة الأصلية إلا بعد إعطاء كلمـة المرور الصحيحة [9].

يحوي نظام التشغيل ويندوز 98 على عدد من حافظات الثاشـة وجميعها تستخدم ملفات صورية نوع BMP. و و. PCX. محدة مسبقاً من قبل النظام و لا تعطي أي طابع شخصي للمستخدم إلا إذا استخدم الحافظة الخاصة بالنص ثلاثي الأبعاد [6].

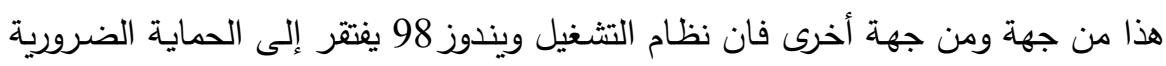

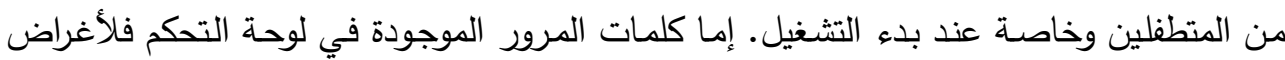
تسجيل الدخول فقط ويمكن تجاوزها بالضغط على المفتاح الموجود في مربع حوار كلمات المرور كما في الثكل (1) [9]. 


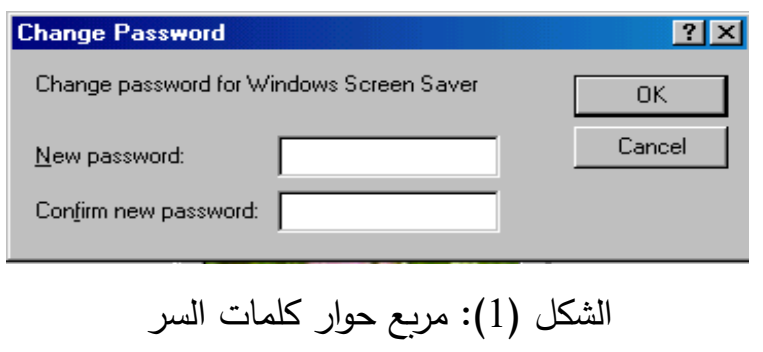

ولإضفاء الطابع الشخصي على الحاسبة لابد من تصميم قالب يكون بمثابة حافظة شاشة

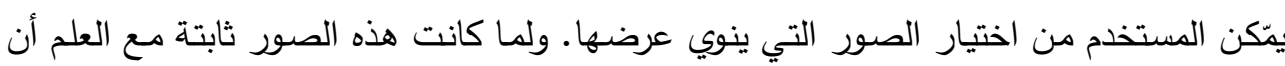

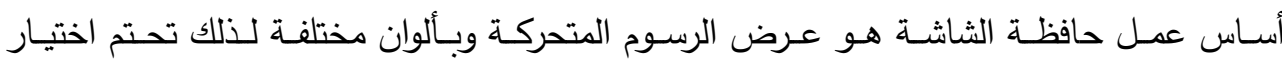

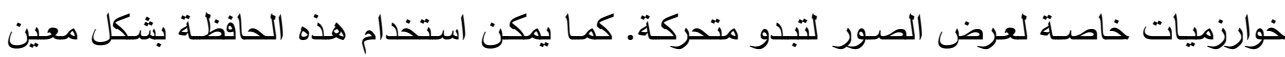

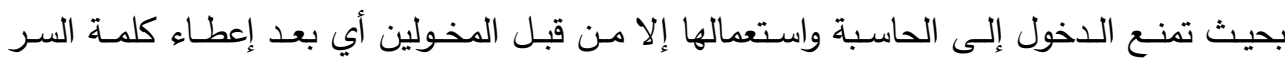

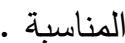

تثبيت برنامج Screen saver .SCR من قبل المستخدم وطريقة إعداده لكي يعمل التطبيق بشكله الصحيح، لابد من وضع الملف (Screensaver) في الداليل

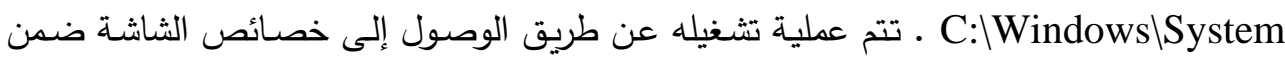

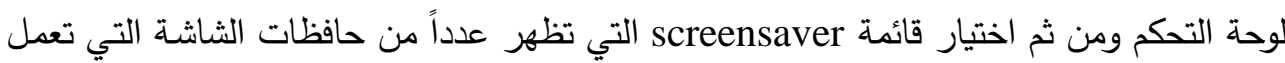
مع ويندوز ومن ضمنها سيظهر اسم البرنامج مع مجموعة الاختيارات كما موجود في الثكل (2).

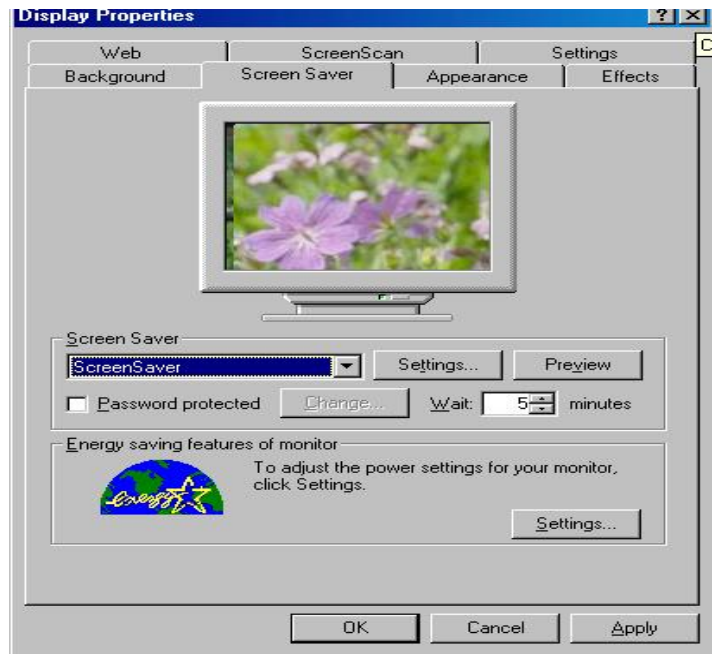

الثكل (2): ظهور التطبيق ضمن اختيارات حافظة الثاشة 
عند الضغط على الإعدادات Settings سيظهر مربع حوار التطبيق، الثكل(3) ويشمل ما يأتي: يستخدم لتحديد سرعة العرض من خلال شريط يمكن تمريره عبر تدرجات : Delay Time

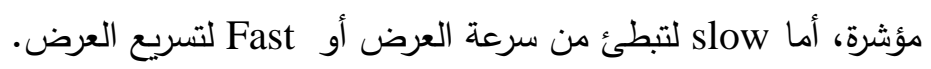
ب- Animation : لتحديد خوارزمية العرض إذ تم توفير خمس خوارزميات للعرض وهي:

Two ، Two Halfs ، Four Sequres Input، Left to Right ، Image Discrete .Halfs Discrete ج- Photo Directory : قد يرغب المستخدم في عرض أية صورة والتي قد لا تتوفر ضمن

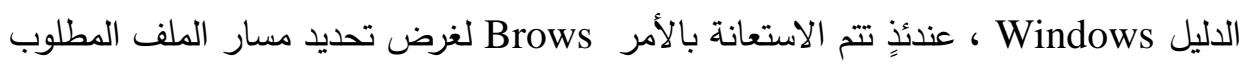

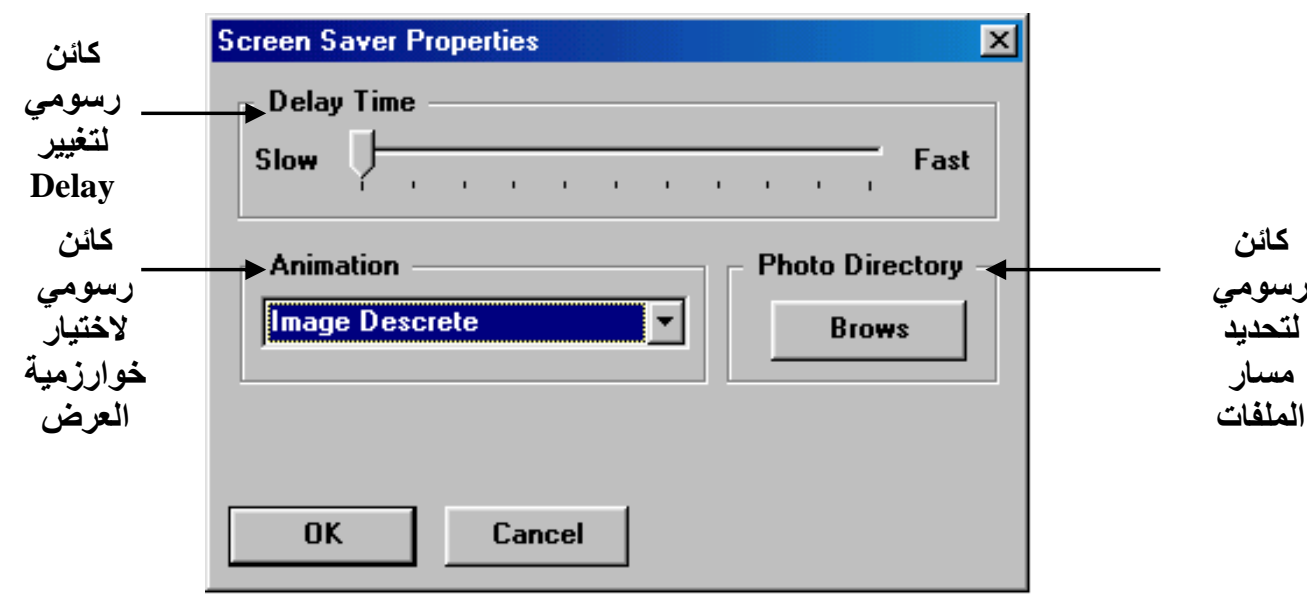

$$
\text { الثكل (3): صندوق حوار التطبيق }
$$

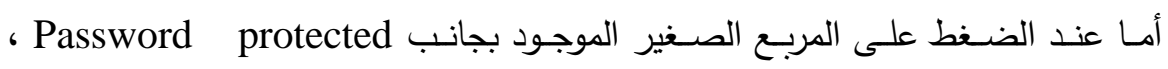

الشكل(2) فسوف يظهر مربع الحوار الذي يطلب إدخال كلمة المرور كما في الثكل (1).

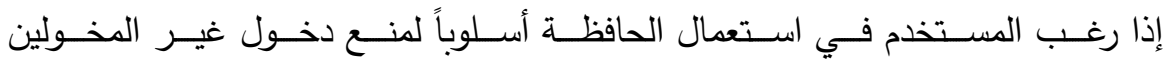

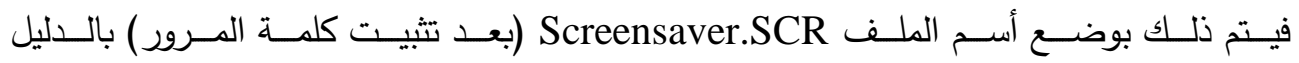
C:IWindows\Allusers \StartMenu\Programs\Startup

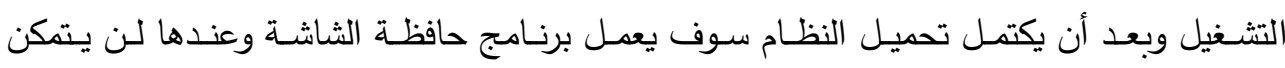
المستخدم من استعمال الحاسبة إلا بعد إعطاء كلمة السر الصحيحة التي سبق خزنها في الإعدادات الموضحة أعلاه. 


\section{ملفات التطبيق}

استخدمت لغة Visual C++ ذات الإصدار 6.0 مـع دوال واجهة البرمجة التطبيقية الـ

ذات البيئة 32-bit ، وهذه اللغة تحتاج إلى ثلاثة أنواع من الملفات لتكوين الملف النهائي

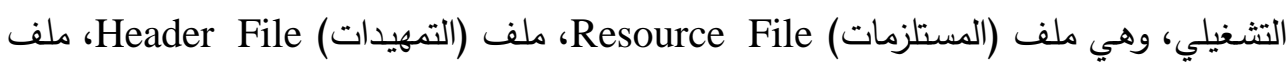
(البرنـامج) Source File. والثكل (4) يبين جميع أجزاء التطبيق من مرحلـة الإنشـاء ولغايـة الحصول على الملف التتفيذي [1][3].

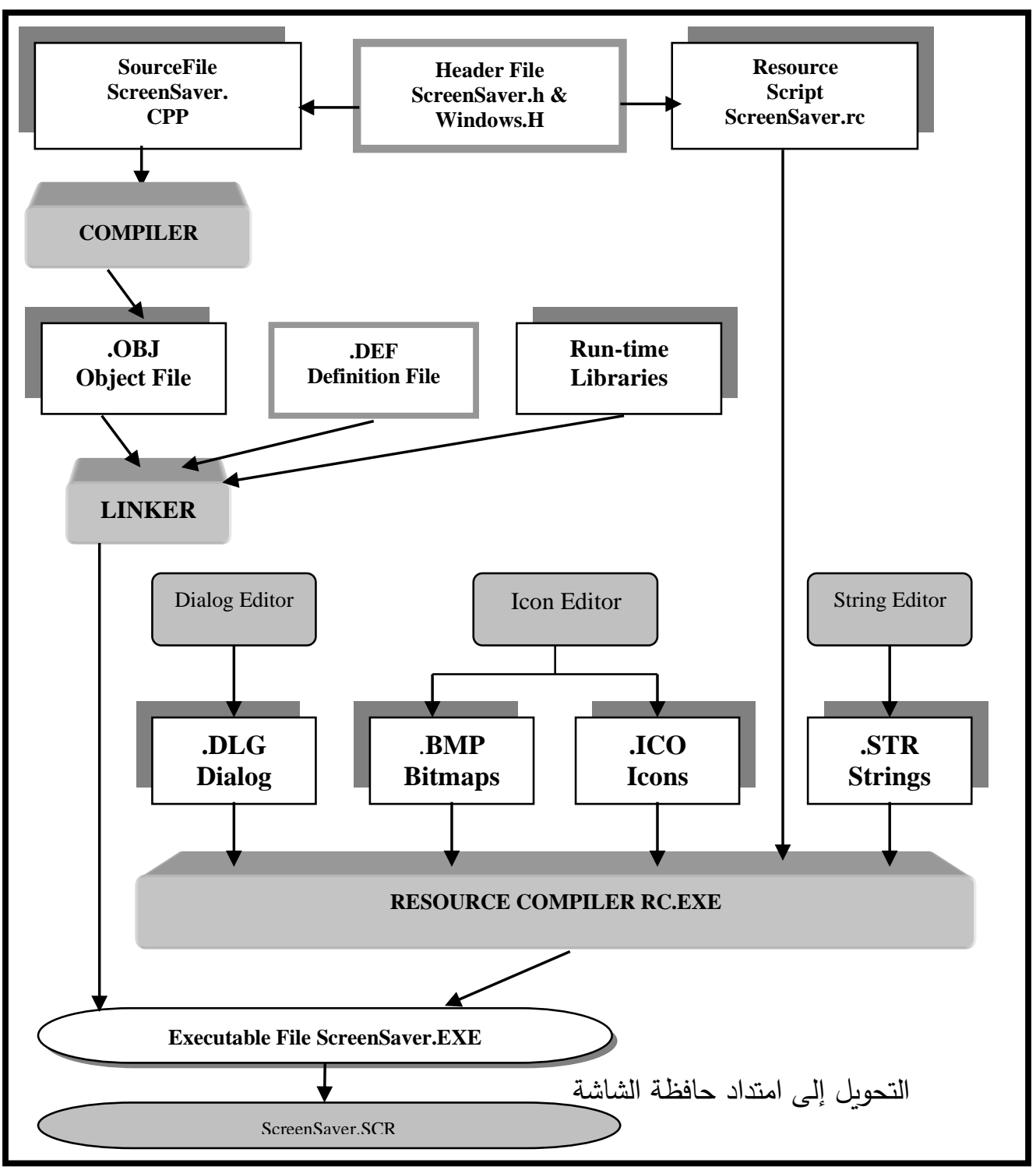

الثكل (4) : تفاعل مكونات التطبيق مع بعضها للحصول على الملف التطبيقي 
أ) ملف المستلزمات ( Resource File )

يصف هذا الملف أنواعاً مختلفة من الكائنات التي تستخدم في ويندوز والتي توضع في

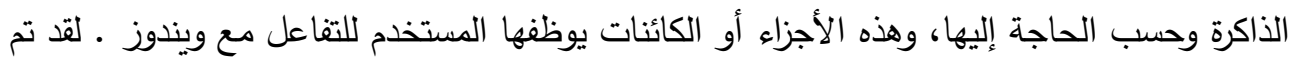

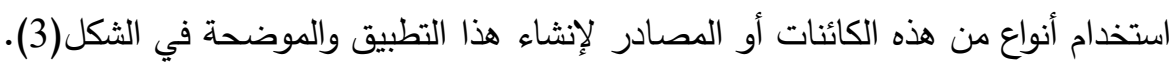

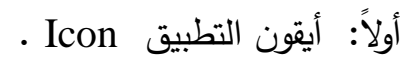

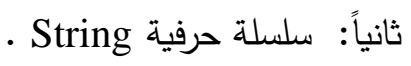

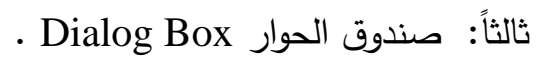
تمثل المتغيرات الموجودة داخل صندوق الحوار (إعدادات التطبيق) والتي على أساسها يكون التثغيل بحيث يتم إعدادها من قبل المستخدم وبما يمثله كل متغير فيها وكالآتي:

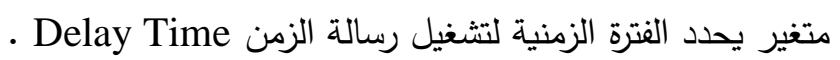

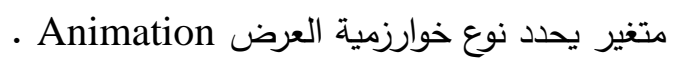

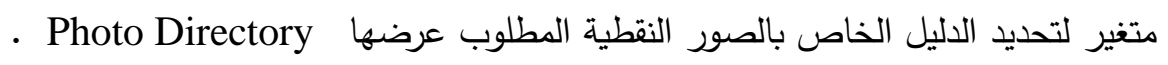

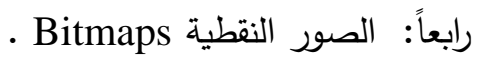

تم استخدام مجموعة من الصور النقطية لغرض العرض، ليس عن طريق ربطها بملف المستلزمات وذلك للحفاظ على التطبيق من ثبات الصور فيه وللتقليل من حجمه، بل يكون ربطها

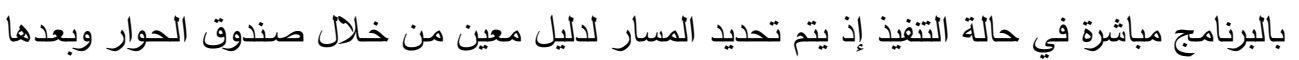

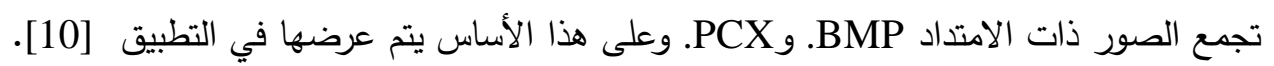

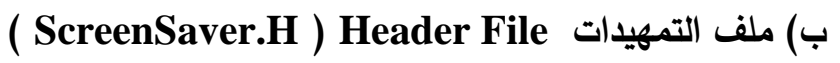
يحوي الأسماء التعريفية لكل الكائنات المستخدمة داخل ملف المستلزمات، إذ أن كل كائن

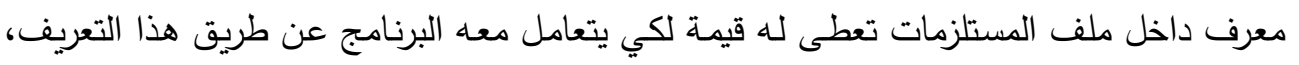

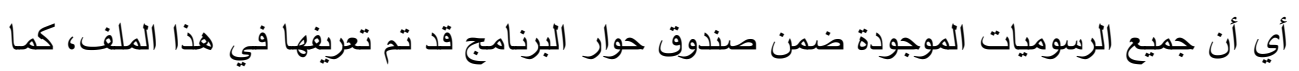

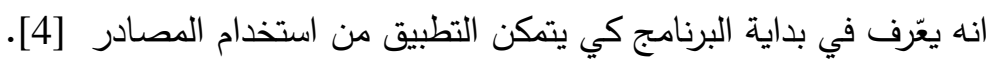
ج) ملف البرنامج الرئيس ( ScreenSaver.CPP )

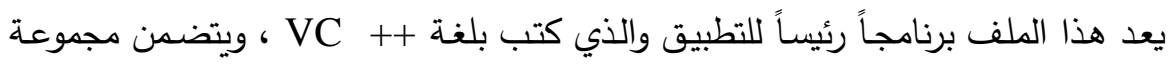

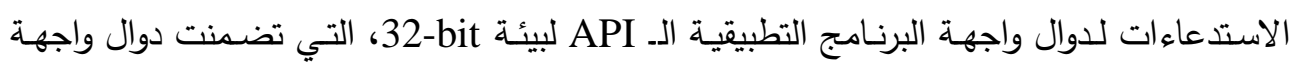

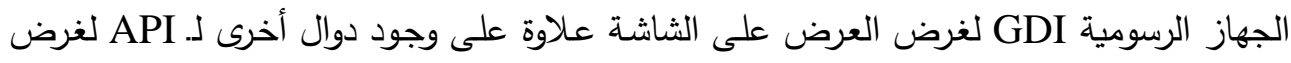

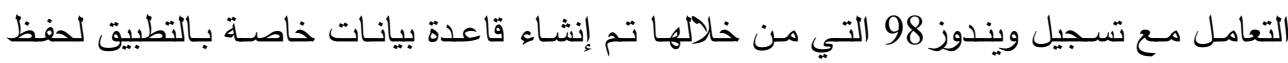
الإعدادات فيها [2] [2] 


\section{إجراءات البرنامج الرئيسة}

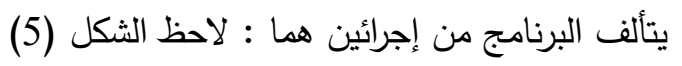

أ) إجراء حافظة الثاشة Screen Saver Proc

يعد هذا الإجـراء القسم التنفيذي للبرنـامج ويعتمـد على دالـة البرنـامج الرئيسـة

Screen SaverProc( )

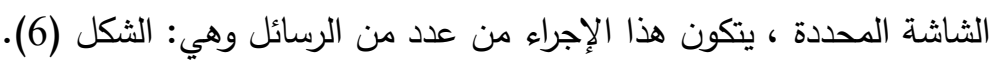

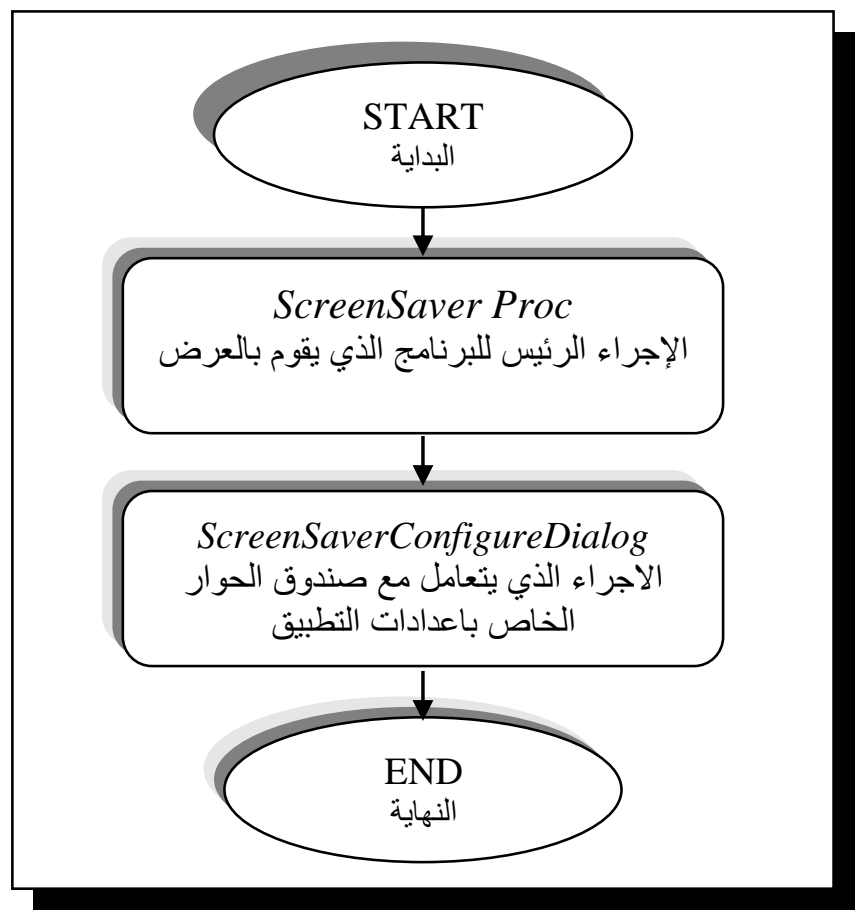

الشكل (5) : أقسام البرنامج الرئيسة 


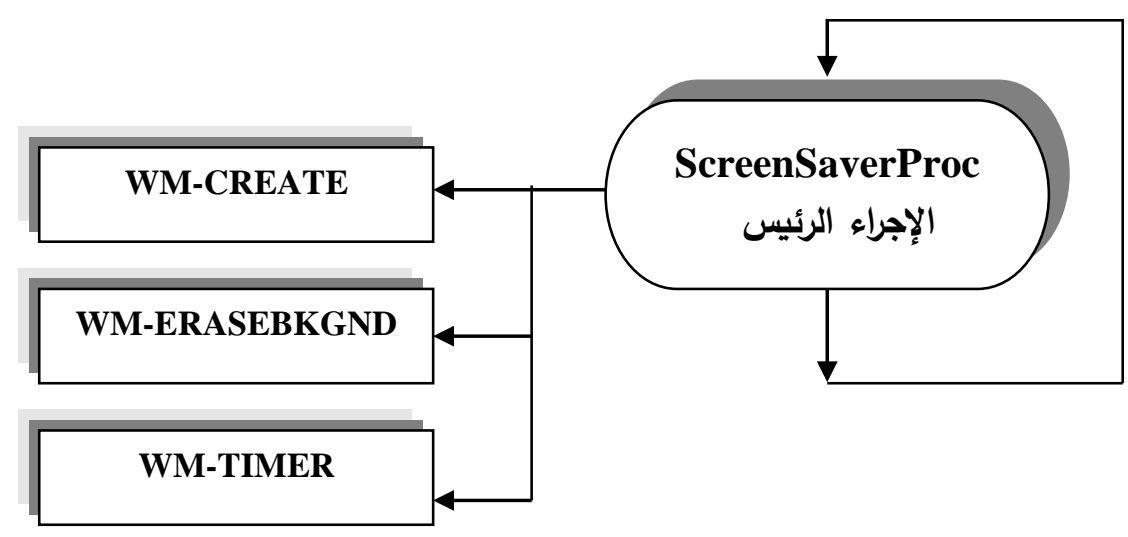

الثكل (6) : أهم الرسائل في الإجراء الرئيس

أولاً: رسالة التكوين WM-CREATE

ترسل هذه الرسالة في حالة استجابة التطبيق لتكوين نافذته إذ يستجيب الإجراء

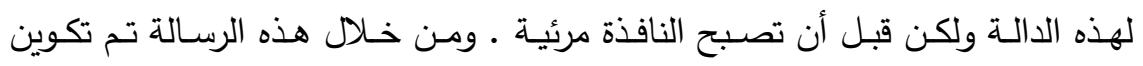

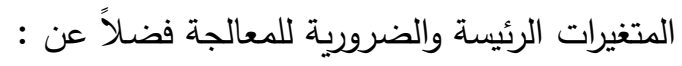

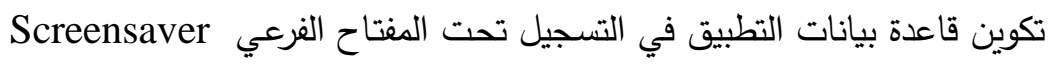

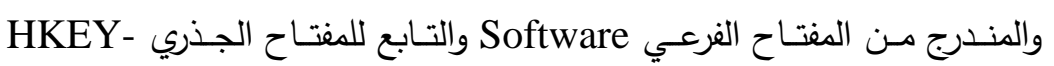

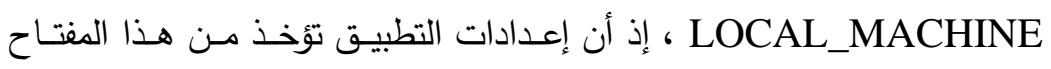

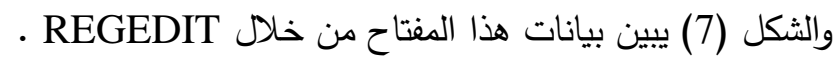

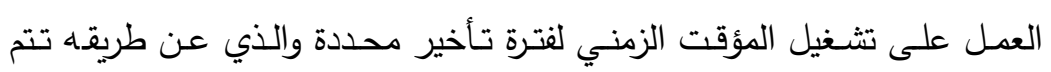

الاستجابة للرسالة الخاصة بالمؤقت ومعالجة الصورة.

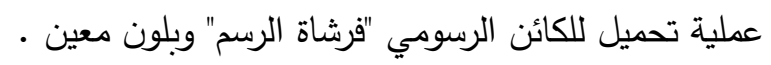

ثانياً: رسالة مسح الخلفية WM-ERASEBKGND

من خلال هذه الرسالة تمت عملية المسح لخلفية النافذة باستخدام فرشاة الرسم

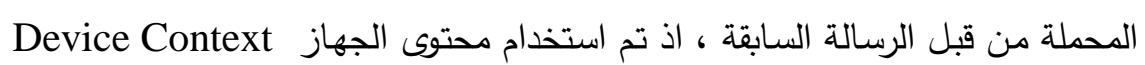

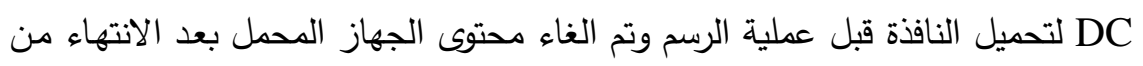

عملية الرسم لخلفية النافذة . 
ثالثاً: رسالة المؤقت او الزمن WM-TIMER

المعالجة لهذه الرسالة وعملية الاستجابة لها هي جوهر البرنامج وتتضمن عملية

العرض الرسومي الصوري بطرق متتوعة. هذه الرسالة يتم ارسالها الى التطبيق خلال فترة

معينة محددة من قبل المستخدم في قاعدة البيانات بعد وضعها في دالة تشغيل الزمن ، وهذه الدالة بدورها تعمل على ارسال هذه الرسالة حسب الفترة المستخدمة فيها وتكون في المتغير Delay ، أي ان كل فترة يتم ارسالها تعني عرضـاً لصورة جديدة بطريقة مختلفة • ويعـى اسـتخدام ويندوز لهذه الرسـالة الى امكانيـة تثبيـت الوقت الذي عنده سـيقطع

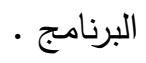

\begin{tabular}{|c|c|c|}
\hline 6 Registry Editor & & $-|\square| x$ \\
\hline Regegistry É dit View $\underline{\text { Help }}$ & & \\
\hline$\square$ HKEY_LOCAL_MACHINE $\Delta$ & Name & Data \\
\hline$\square$ Config & ab] (Default) & [value not set] \\
\hline$\square$ Enum & 임 chosse & $0 \times 00000006(6)$ \\
\hline † Hardware & 임영 delay & $0 \times 00000002$ (2) \\
\hline Network & ab] message & "Screen Saver Application" \\
\hline Software & abjpath & "D: \\
\hline $\begin{array}{l}+\square \text { ACD Systems } \\
+\square \text { Adobe }\end{array}$ & & \\
\hline$\square$ Borland & & \\
\hline$\square$ CLASSES & & \\
\hline (1) Clients & & \\
\hline$\square$ Description & & \\
\hline Dream Group 1998-1 & & \\
\hline INTEL & & \\
\hline$\square$ K.S.Waves & & \\
\hline Yodak & & \\
\hline McAfee & & \\
\hline Network Associates & & \\
\hline$\square 0 D B C$ & & \\
\hline Policies & & \\
\hline$+\mathrm{scC}$ & & \\
\hline$=$ & 11 & . \\
\hline NHKEY LOCAL MACHINEIS & 1 & \\
\hline
\end{tabular}

الشكل (7) : بيانات المفتاح screensaver داخل التسجيل

هنالك متطلبات يجب توفيرها أو إجراؤها لكي تتم المعالجة الصورية وهي:

تحميل محتوى جهاز للذاكرة .

تعريف هيكل للصورة النقطية لاخذ معلومات الصورة منها .

تحميل الصورة في الهيكل المعرف . ت 


$$
\begin{aligned}
& \text { 口 تحديد او الحصول على الكائن الرسومي المستخدم . }
\end{aligned}
$$

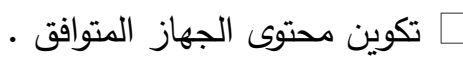

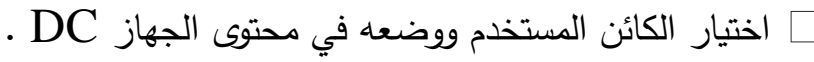

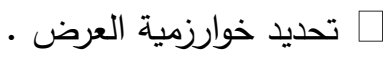

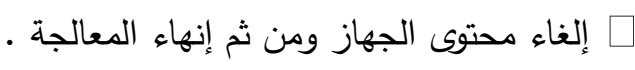

وتجدر الإشارة هنا إلى انه قبل خوارزمية العرض لابد من الحصول على على الصورة النقطية

ضمن المسار المحدد لعدد من الملفات الموجودة في دليل ما ومن ثم عرضها الواحدة تلو الأخرى. وعن طريق المتغير الموجود في صندوق الحوار Brows يتم تحديد المسار المطلوب ، لاحظ

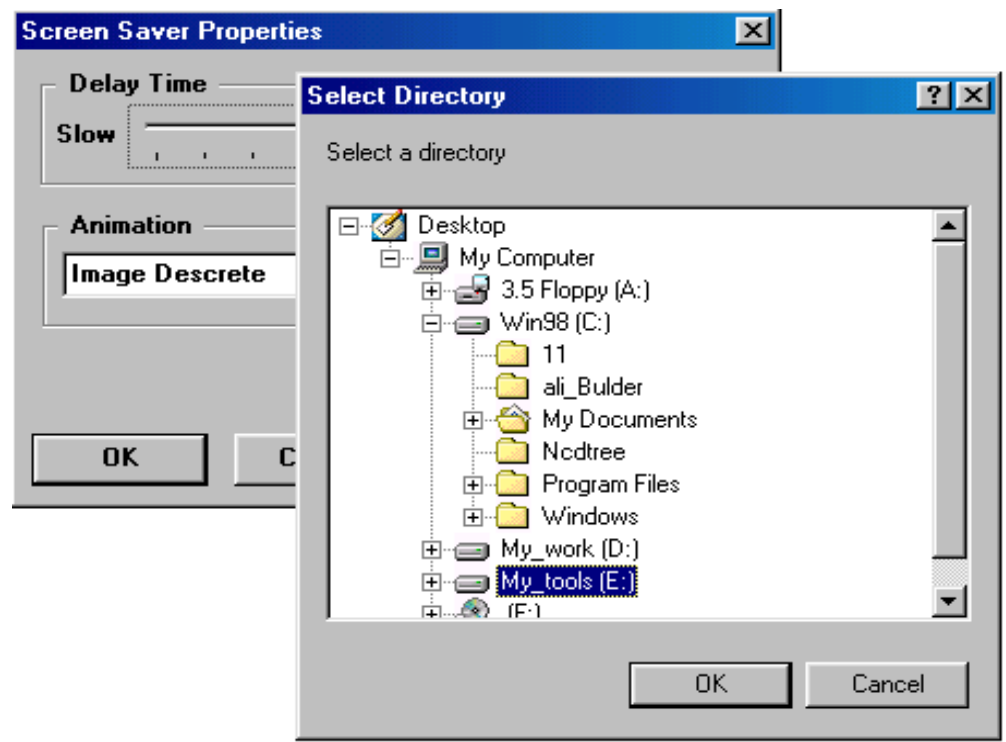

$$
\text { الثكل (8) : تحديد دليل الملفات الصورية }
$$

بعد الانتهاء من هذه العمليات والتي عن طريقها تم التعامل مع واجهة الجهاز الرسومية، سيتم تحديد الخوارزمية المطلوبة حسب طلب المستخدم الذي سبق خزنه في المتغير chose ضمن التسجيل ـ الخوارزميات المتوفرة هي (الثكل 9): 


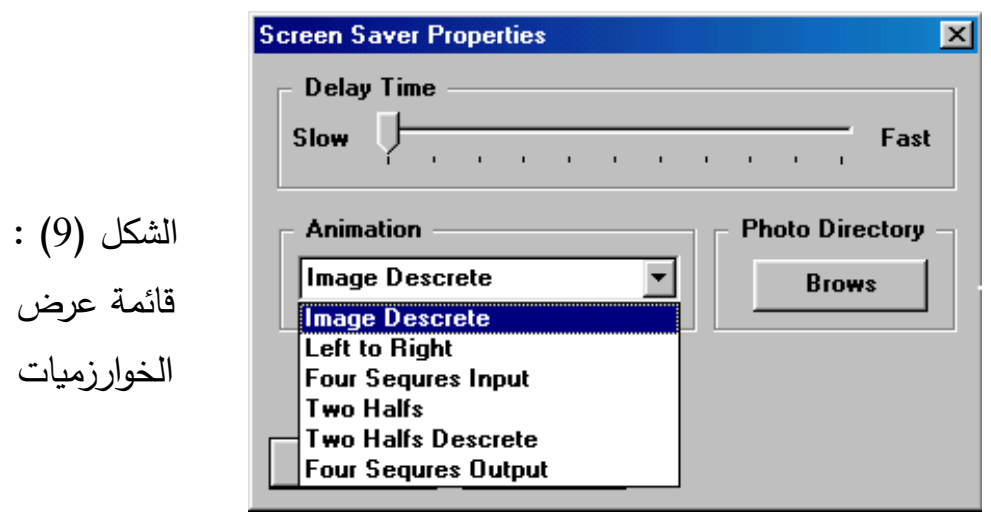

أولاً: خوارزمية ( Image Discrete ) ، عملية عرض للصورة تتم بأخذ بياناتها من محتوى الجهاز

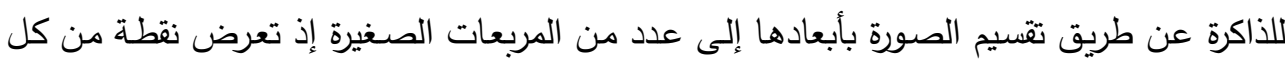

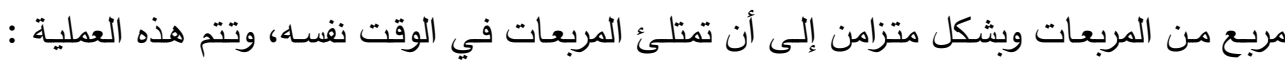
لاحظ الملحق (1)

$$
\begin{aligned}
& \text { تحديد المربعات المطلوبة لتقسيم الثاشة اعتماداً على حجم الصورة. } \\
& \text { اخذ البيانات من الصورة ووضعها في المواقع المحدة للمربعات . } \\
& \text { وضع البيانات على الثاشة نقطة نقطة. }
\end{aligned}
$$

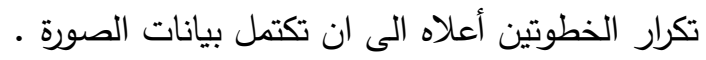

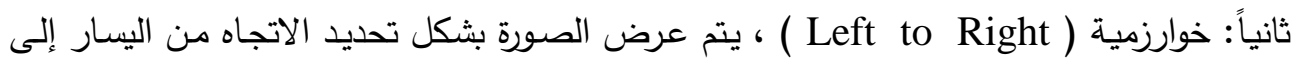

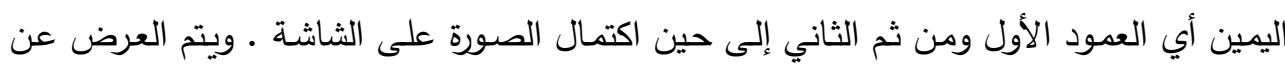
طريق : الملحق (2).

$$
\begin{aligned}
& \text { تثييت المحور العمودي للصورة . } \\
& \text { التغيير يكون عن طريق المحور الأفقي . } \\
& \text { عرض كل نقطة للصورة. }
\end{aligned}
$$

ثالثاً: خوارزمية ( Four Sequres Input )، تقسيم الصورة إلى أربعة مربعات متساوية ويكون

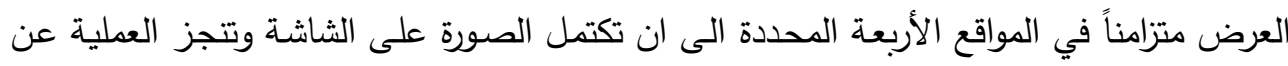

طريق : الملحق (3).

تثبيت النقطة الأولية لكل مربع والمأخوذ من بيانات الصورة .

تكوين دارة معينة وبعدد معين لا يتجاوز أبعاد الصورة ثم يتم العرض لكل لكل نقطة

$$
\text { في المربعات المحددة وفي الوقت نفسه. }
$$


رابعاً: خوارزمية ( Two Halfs ) ، تكون صيغة العرض بتحديد عمود من جهة اليسار و أخر من

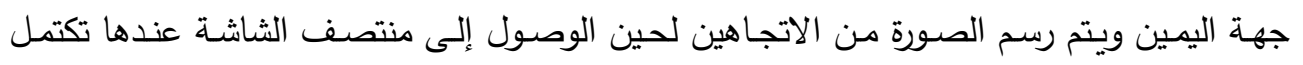
الصورة، الملحق (4).

خامساً: خوارزمية ( Two Halfs Discrete ) ، يتم العرض فيها عن طريق تكوين خطوط أفقية تبدا من اليسار والأخرى من جهة اليمين ولكن ليست على المستوى نفسه ويستمر العرض إلى حين

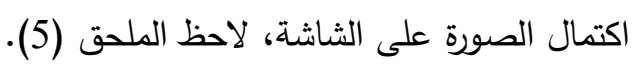
وهكذا فان الصورة المستخدمة في الخوارزميات يتم الحصول عليها من خلاد دالة البحث وذلك في المسار المأخوذ من قاعدة البيانات ، وعند عرضها يتم البحث عن أخرى وهكذا دواليك . إن العملية الأخيرة التي يجب القيام بها هي إلغاء الكائن الرسومي وإلغاء لمحتوى الأجهزة المعرفة وبعد ذلك يتم الانتهاء من الإجراء الرئيس للبرنامج •

\section{Screen Saver Configure Dialog}

ب) (إجراء صندوق الحوار

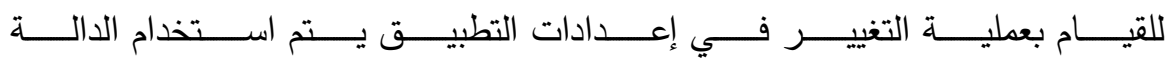

ScreenSaverConfigureDialog( )

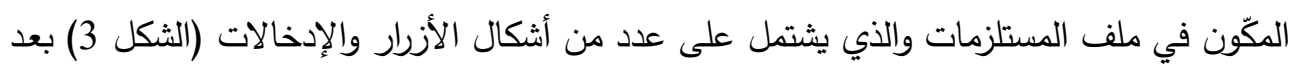

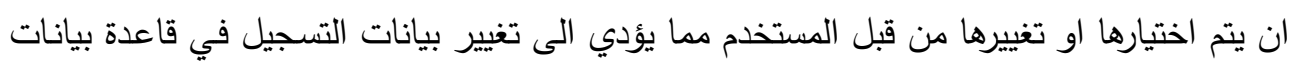

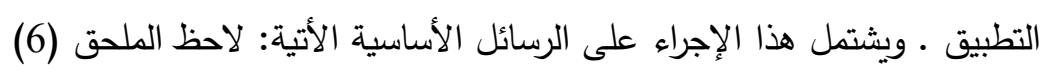

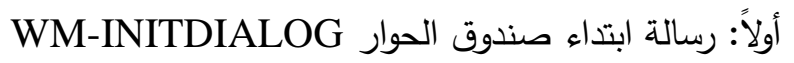

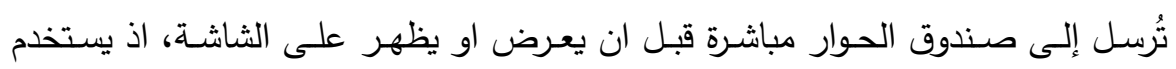

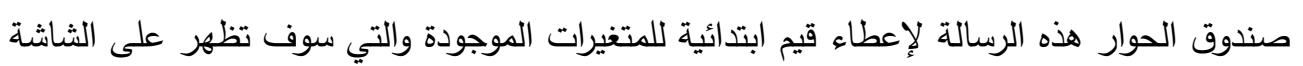

ومن خلال هذه الرسالة تمت المعالجة الأتية على صندوق الحوار :

تكوين بعض الكائنات الرسومية التابعة لصندوق الحوار والتي لم نستطع تكوينها

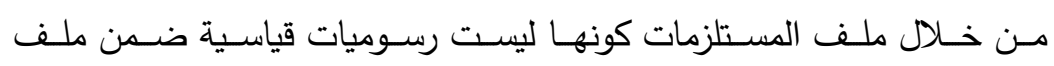

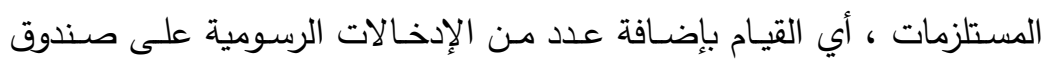

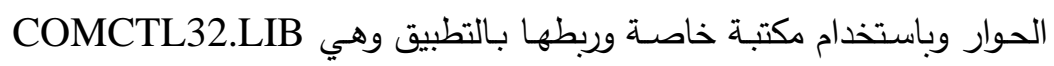

فضلاً عن الملف التمهيدي COMMCTRL.h. 
عمليـة إظهـار إعـادات التطبيـق في صـندوق الحـوار عـن طريـق اخــ هـــه

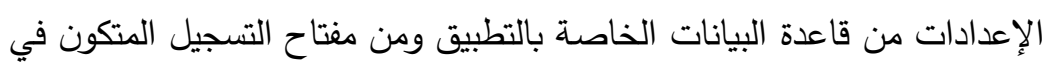

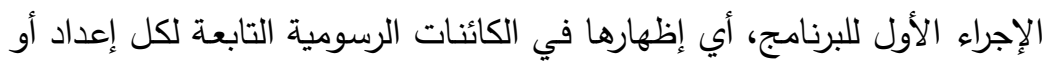
متغير

وصندوق الحوار هنـا يمكن المستخدم الإبقاء على إعدادات التطبيق أو تغييرهـا حسب الرغبة .

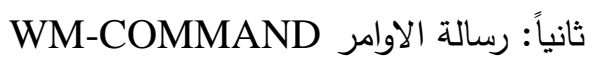

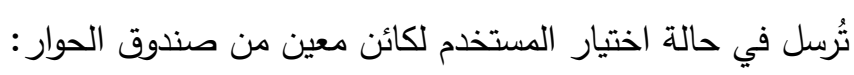
Brows

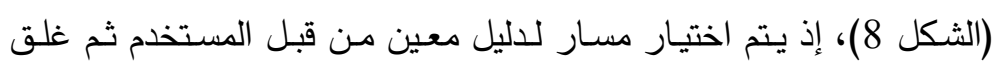

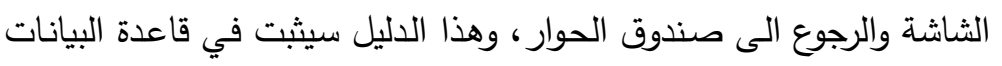

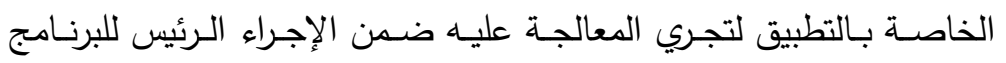

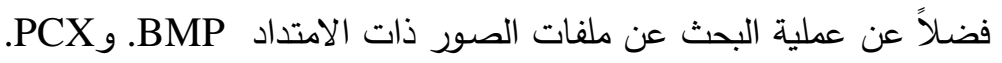
ليتم عرضها في التطبيق • Ok Ok

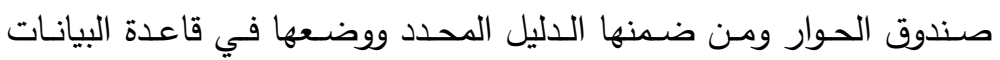
الخاصة بالتطبيق ، ثم غلق صندوق الحوار . Cancel

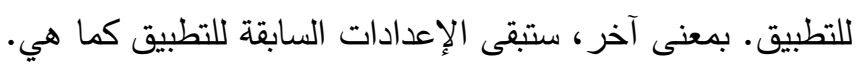

التطبيق (ScreenSaver) هو قالب لحافظة شاشـة يمكن أن تستخدم فيه أيـة صـورة

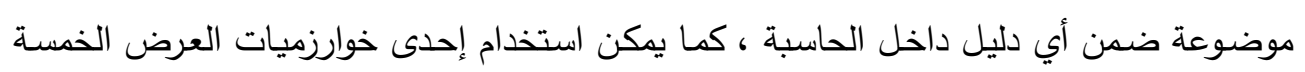

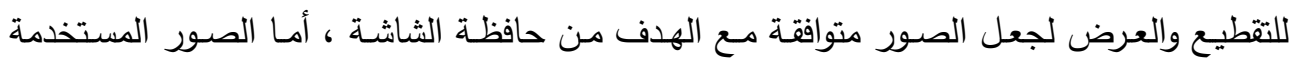
فيجب ان تحمل الامتداد BMP. و.PCX. وهي الأنواع المعتمدة في ويندوز ـ كما يمكن استخدام

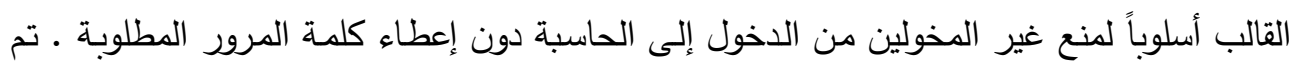

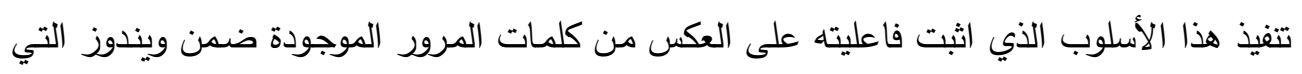
غالباً ما يتم تجاوزها بأي شكل من الأشكال. 


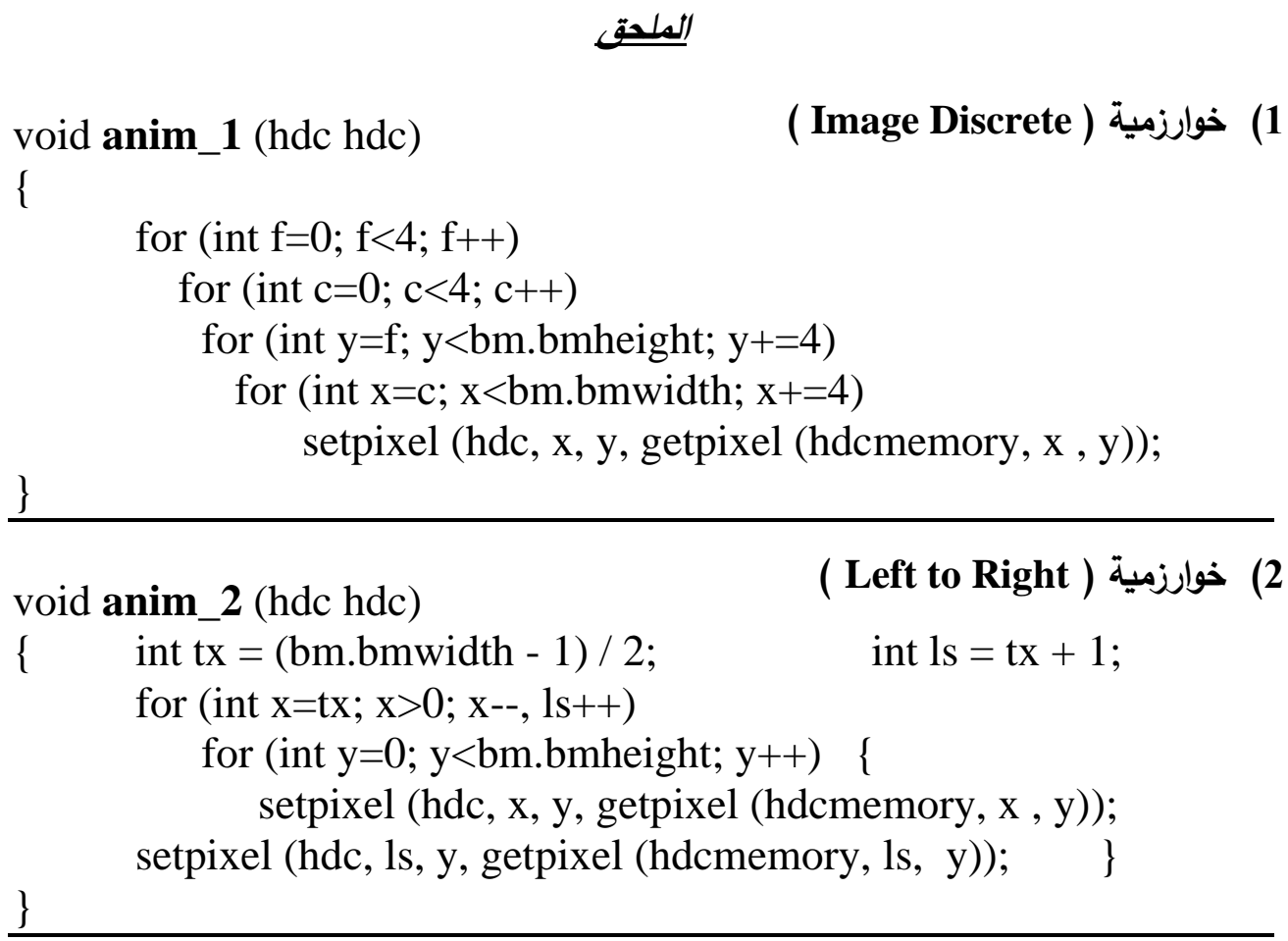

void anim_3 (hdc hdc)

\{ int ls = bm.bmwidth-1;
(3) خوارزمية ( Four Sequres Input )

$$
\text { int } \mathrm{tx}=1 \mathrm{~s} / 2 \text {; }
$$

for (int $\mathrm{x}=0$; ls $>\mathrm{tx} ; \mathrm{x}++, \mathrm{ls}--$ )

for (int $\mathrm{y}=0 ; \mathrm{y}<\mathrm{bm}$. bmheight; $\mathrm{y}++$ ) \{

setpixel (hdc, $\mathrm{x}, \mathrm{y}$, getpixel (hdcmemory, $\mathrm{x}, \mathrm{y})$ );

setpixel (hdc, ls, y, getpixel (hdcmemory, ls, y))

$\underline{3}$

void anim_4 (hdc hdc)

(4) خوارزمية ( Two Halfs)

$\{$ int $\mathrm{i}, \mathrm{j}, \mathrm{k}, \mathrm{l}$;

setpixel (hdc, x1, y1, getpixel (hdcmemory, x1, y1));

setpixel (hdc, x2, y2, getpixel (hdcmemory, x2, y2));

setpixel (hdc, x3, y3, getpixel (hdcmemory, x3, y3));

setpixel (hdc, x4, y4, getpixel (hdcmemory, $\mathrm{x} 4, \mathrm{y} 4)$ );

for $(\mathrm{i}=1 ; \mathrm{i}<\mathrm{y} 1+1 ; \mathrm{i}++) \quad\{\quad \mathrm{l}=\mathrm{i}$;

for $(\mathrm{k}=1 ; \mathrm{k}<3 ; \mathrm{k}++)$

for $(j=x 1-i ; j<x 1+i+1 ; j++)$

setpixel (hdc, j, y1-1, getpixel (hdcmemory, j, y1-1)); 
setpixel (hdc, j+x $1 * 2$, y1-1, getpixel (hdcmemory, j+x1*2, y1-1)); setpixel (hdc, j, y1*3-1, getpixel (hdcmemory, j, y1*3-1));

setpixel (hdc, $\mathrm{j}+\mathrm{x} 1 * 2$, y3-1, getpixel (hdcmemory, $\mathrm{j}+\mathrm{x} 1 * 2, \mathrm{y} 3-1)$ );

\}

$$
\text { \} } 1=1 *-1 ; \quad\}
$$

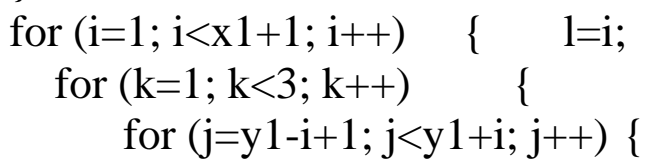

setpixel (hdc, $x 1 * 3+1, j+y 2 * 2$, getpixel (hdcmemory, $x 1 * 3+1$,

$$
1=1 *-1 ; \quad\} \quad\}
$$

\}

void anim_5 (hdc hdc)

(5wo Halfs Discrete ( خوارمية )

$\{$ int ls = bm.bmwidth-1; int $\mathrm{tx}=1 \mathrm{~s} / 2$;

for (int $\mathrm{x}=0 ; 1 \mathrm{~s}>0 ; \mathrm{x}++, 1 \mathrm{~s}--)$

for (int $\mathrm{y}=0 ; \mathrm{y}<\mathrm{bm} . \mathrm{bmheight} ; \mathrm{y}+=2) \quad\{$

setpixel (hdc, $x, y$, getpixel (hdcmemory, $x, y)$ );

setpixel (hdc, ls, y+1, getpixel (hdcmemory, ls, y)); \}

\}

6creenSaverConfigureDialog إجراء صندوق الحوار

bool winapi screensaverconfiguredialog

(hwnd hdlg, uint message, wparam wparam, lparam lparam)

\{

switch (message) \{

case wm_initdialog:

initcommoncontrols (); isok = true;

regcreatekeyex (hkey_local_machine, "softwarellscreen_saver",

0, "screen saver", 0, key_all_access, null, \&hregkey, \&result);

regqueryvalueex (hregkey, "delay", null, \&datatype, null,

\&datasize);

regqueryvalueex (hregkey, "delay", null, \&datatype, lpbyte) 
\&delay, \&datasize);

regqueryvalueex (hregkey, "chosse", null, \&datatype, null,

\&datasize);

regqueryvalueex (hregkey, "chosse", null, \&datatype, (lpbyte)

\&chosse, \&datasize);

regqueryvalueex (hregkey, "path", null, \&datatype, null,

\&datasize);

regqueryvalueex (hregkey, "path", null, \&datatype, (lpbyte)

\&path, \&datasize);

hwndslider = createwindow (trackbar_class, "", ws_child|ws_visible|tbs_autoticks,50,30,240,25,hdlg,null,hd, null); sendmessage (hwndslider, tbm_setrange, (wparam)1, (lparam) makelong $(0,100))$;

sendmessage (hwndslider, tbm_setpos, (wparam)true, (lparam) (delay/10));

senddlgitemmessage (hdlg, idc_combo1, cb_addstring, 0 , (lparam) "image descrete");

senddlgitemmessage (hdlg, idc_combo1, cb_addstring, 0 , (lparam) "left to right");

senddlgitemmessage (hdlg, idc_combo1, cb_addstring, 0 , (lparam) "four sequres");

senddlgitemmessage (hdlg, idc_combo1, cb_addstring, 0 , (lparam) "two halfs");

senddlgitemmessage (hdlg, idc_combo1, cb_addstring, 0 , (lparam) "two halfsdescrete");

senddlgitemmessage (hdlg, idc_combo1, cb_addstring, 0 , (lparam) "zzzzzzzzzzzzz");

senddlgitemmessage (hdlg, idc_combo1, cb_setcursel, (((chosse $>=1) \& \&($ chosse $<=6))$ ? chosse $-1: 0), 0)$; return true;

case wm_command:

switch (loword (wparam))

case idset: showbrowse (hdlg); isok $=$ showfile $\left(" \backslash *^{*}\right.$. bmp"); break;

case idok:

if (!isok) $\quad\{$ killtimer (htimer, 1); else \{ messagebox (null, msg, "warring", 0); \} 
$\mathrm{sp}=$ sendmessage $($ hwndslider, tbm_getpos, $($ wparam $) 0$,

killtimer (htimer, 1); $\quad$ delay $=\mathrm{sp} * 10$;

(lparam)0);

timer $=$ settimer (htimer, 1 , delay, null);

chosse $=$ senddlgitemmessage $($ hdlg, idc_combo1, b_getcursel, $0,0)+1$

if $($ chosse $==0) \quad$ chosse $=1$;

regsetvalueex (hregkey, "delay", 0, reg_dword, (lpbyte)

\&delay, sizeof (dword));

regsetvalueex (hregkey, "chosse", 0, reg_dword, lpbyte)

\&chosse, sizeof (dword));

regsetvalueex (hregkey, "path", 0, reg_sz, (lpbyte)path, strlen

(path)+1);

\}

regclosekey (hregkey); enddialog (hdlg, 0); break;

case idcancel: $\quad$ enddialog $(\mathrm{hdlg}, 0)$; return true;

\} return true;

\} return false;

\} 
المصادر

[1] Andrews, M., 1996, "Learn Visual C ++ Now", Ms Press.

[2] Myers, B. \& Doner, C., 1988, “ Graphics Programming under Windows", SYBEX Inc.

[3] Pappas, C., H. \& Murray, W., H., 1997, The Complete Reference Visual C++ 5, McGraw-Hill Companies.

[4] Petzold, C., 1992, Programming Windows 3.1, $3^{\text {td }}$ Edition, Microsoft Press.

[5] Randall, N., 2000, "Give Your Desktop a Makeover: Computer Programs", PC Magazine,Vol.19, Issue6, P.104.

[6] Rubenking, N.J, 2000, "Mutate your Screen: Screen Savers", PC Magazine, Vol. 19, Issue 16, , P. 117.

[7] Schildt, H.,1998, Windows 98 Programming from the GROUND UP, McGraw-Hill Companies.

[8] Steers, K., 1999, “Troubleshooting: Screen Saver”, PC World, Vol. 17, No. 8, P.136.

[9] Steers, K., 2000, "Windows System Monitor Peeks Under a PC Hood : Screen Savers", PC World, Vol. 18, Issue 11.

[10] Yao, P. \& Leinecher, R., C., 1997, "Visual C++ 5 Bible", IDG Books World Wide Inc. 